\title{
Graphene Electron States in a Quantum Well
}

\author{
V.A. Fedirko \\ Moscow State University of Technology "Stankin" (MGTU "Stankin"), Russia \\ National Research University of Electronic Technology "MIET” (MIET), Russia
}

Copyright $(\mathcal{C} 2015$ by authors, all rights reserved. Authors agree that this article remains permanently open access under the terms of the Creative Commons Attribution License 4.0 International License.

\begin{abstract}
The paper reports on theoretical estimation for electron spectra of free-standing graphene monolayer in presence of a rectangular quantum well. We have shown that 1D quasi-localized states localized mainly in the quantum well may exist, forming a peculiar quantum "rod" in a grapheme monolayer. Conventional hole tunneling through the quantum well is also described in addition to Klein tunneling usually considered in such structure.
\end{abstract}

Keywords Graphene, Quantum Well, Quantum Tunneling, Quasi-localized States

\section{Introduction}

Since the first report of successful fabrication by pulling out of atomic monolayer out of bulk graphite and its description [1,2] graphene has been attracting unfading interest as a fascinating system for fundamental studies and a promising candidate material for various future applications [3] (see also the review article [4]). Using technological tailoring or electric field a potential relief can be created in a graphene layer which gives new opportunities for its application.

Graphene is a mono-atomic layer of carbon atoms arranged on a 2D honeycomb lattice. Near each corner of the hexagonal first Brillouin zone (also called Dirac points or $\boldsymbol{K}$-points) the quasiparticle excitations obey a 2D linear dispersion relation and behave like massless "relativistic" particles. That leads to a number of unusual electronic properties; one of them is so called Klein paradox [5] well known in quantum electrodynamics. It predicts that the electron can perfectly pass through the potential barrier independently of its height in contrast to the conventional nonrelativistic tunneling where the transmission probability exponentially decays with the barrier height increasing. As was shown in $[6,7]$ the similar phenomenon takes place in graphene (see also [8]). So some electron states in graphene with a quantum well (which can be considered as a barrier for holes) are those corresponding just to that Klein (or "chiral") tunneling in both directions. Klein chiral tunneling in graphene has been further studied by various authors (see, e. g., [9-11]). However, chiral tunneling states, though quite essential, are far from exhausting the whole spectrum of graphene electron-hole excitations in presence of a quantum well (or a quantum barrier). In that paper we make an attempt to study all variety of allowed states in such a system, using a rectangular quantum well $(\mathrm{QW})$ as a model potential relief. The detailed theoretical study of electron spectrum even in such a simple structure has not been performed yet. Underscore that we consider the QW inside formally unbounded graphene layer, not touching upon tunneling out from graphene through its edge [12].

\section{Basic Premises}

Electron excitations in graphene near Dirac points are described by two-component wave functions $\psi(x . y)=\left[\varphi_{A}, \varphi_{B}\right]$ which are the envelope amplitude on sublattices $A$ and $\mathrm{B}$ of graphene honeycomb lattice (Fig. 1). They obey 2D Dirac-Weyl-like equation [8, 13-15]:

$$
\hat{H} \psi=\left\{-i \hbar v_{0} \sigma \cdot \nabla+V \cdot I\right\} \psi=E \psi,
$$

where $v_{0} \approx 10^{8} \mathrm{~cm} / \mathrm{c}$ is the characteristic velocity (called Fermi velocity by some authors $[7,8]), \sigma=\left(\sigma_{x}, \sigma_{y}\right)$ are the Pauli matrices, $I$ is the $2 \times 2$ unit matrix, $\nabla=(\partial / \partial x, \partial / \partial y), V$ is the potential energy.

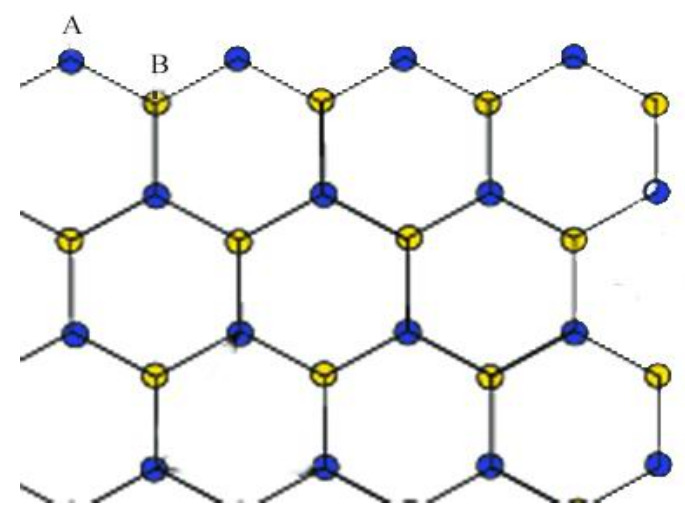

Figure 1. A fragment of a graphene lattice 
Assuming the potential energy only $x$-dependent, the wave functions, both for sublattice $A$ and $B$, can be written in the form:

$$
\varphi_{A, B}(x, y)=\varphi_{A, B}(x) \cdot e^{i k y}
$$

We let the potential $V$ be a square quantum well (QW) of finite width $a$ and depth $U$, and limit our consideration to the most interesting electron energies $0>E>U$.

Introduce the notations: $\kappa>0, \bar{\kappa}>0, u>0$ and $\mu>0$ by the relations:

$E=-\hbar v_{0} \kappa ; U=-\hbar u / a ; E-U=\hbar v_{0} \bar{\kappa} ; u / v_{0}=|U| a / \hbar v_{0}=\mu$ ( $\hbar u=\int|U| d x \sim|U| \cdot a$ is sometimes called the "power" of a potential well). It is also convenient to use normalized values by

$$
k_{0}=\mu / a=|U| / \hbar v_{0},
$$

e.g., $k \rightarrow k / k_{0}, \kappa \rightarrow \kappa / k_{0}$, etc., with normalized $k$ and $\kappa$ in the interval $0 \leq(k, \kappa) \leq 1$.

The dimensionless $(\kappa, k)$-domain is naturally fragmented into 4 sectors as shown in Fig. 2 as the electron energy spectrum in them is utterly different.

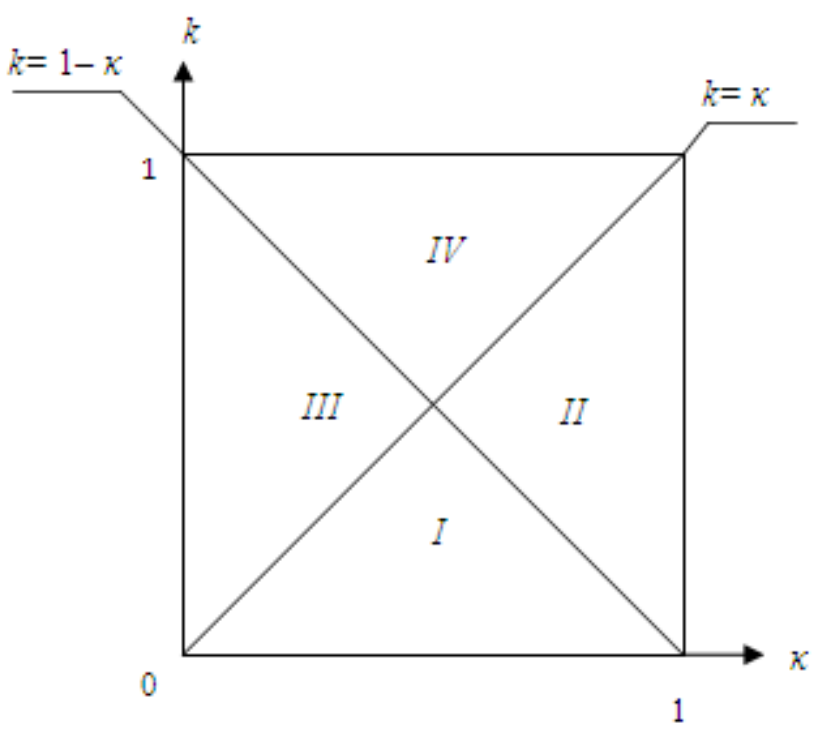

Figure 2. Specific sectors of dimensionless $(\kappa, k)$-domain

Below we consider the boundary problem for Eq. 1 arising for elementary excitation stationary states in each of those sectors.

\section{Excitations Spectrum}

\section{In sector I of Fig.2:}

$$
0 \leq \kappa \leq 1, \mathrm{k} \leq 1 / 2
$$

- the abovementioned chiral tunneling (Klein tunneling) takes place. We remind briefly its main points referring to [6] for details.

Eq. (1) is satisfied by the set of wave functions:

$$
\begin{aligned}
& \varphi_{A}(x)=\frac{1}{\sqrt{2}} \cdot \begin{cases}e^{i p x}+r e^{-i p x}, \quad x<0 \\
b e^{i q x}+c e^{-i q x}, \quad 0<x<a \\
t e^{i p x}, \quad x>a\end{cases} \\
& \varphi_{B}(x)=\frac{1}{\sqrt{2}} \cdot\left\{\begin{array}{l}
-\frac{1}{\kappa}\left[(p+i k) e^{-i p x}+r(p-i k) e^{i p x}\right], \quad x<0 \\
+\frac{1}{\bar{\kappa}}\left[(q+i k) b e^{i q x}-(q-i k) c e^{-i q x}\right], \quad 0<x<a \\
-\frac{(p+i k)}{\kappa} t e^{i p(x-a)}, \quad x>a
\end{array}\right.
\end{aligned}
$$

where

$$
p=\sqrt{\kappa^{2}-k^{2}}, \bar{\kappa}=\sqrt{q^{2}+k^{2}}=(E-U) / \hbar v_{0}
$$

or - in dimensionless variables

$$
\bar{\kappa}=\sqrt{q^{2}+k^{2}}=1-\kappa .
$$

The coefficients $r, b, c$ and $t$ are found by matching boundary conditions at $x=0, a$ for any $k$ and $\kappa$ in that sector.

Since the electron velocity is directed opposite to the wave vector for $E<0$, the incident and transmitted waves may be interpreted as incident and transmitted hole flews respectively, while inside the well the resulting electron flew is formed when $E>U$, as shown in Fig. 3.

As was shown in [6-8] the transmission $T=|t|^{2}$ in that case does not decay exponentially with $a$ increasing, and may reach resonant value $T=1$ for some incident angles and when the condition $q a=\pi n$ is realized with an integer $n$, which is just the peculiar feature of Klein tunneling. We however draw one's attention to the essential fact that such chiral tunneling is possible only if the $y$-component of an electron wave vector $k<k_{0} / 2=|U| / 2 \hbar v_{0} \quad(k<1 / 2$ in dimensionless variables, see Fig. 2); the maximum $k=k_{0} / 2=|U| / 2 \hbar v_{0}$ is allowed only for $\kappa=k_{0} / 2=|U| / 2 \hbar v_{0}$ - that is for electron energy $E=U / 2$, but the tunneling probability in that case is equal to zero as the electron wave vector is parallel to the barrier border. When $0>E>U / 2$ chiral tunneling takes place for any incident angle of the electron wave to the barrier border $0<\theta<\pi / 2$, but when $U / 2>E>U$ chiral tunneling is possible only if the incident angle:

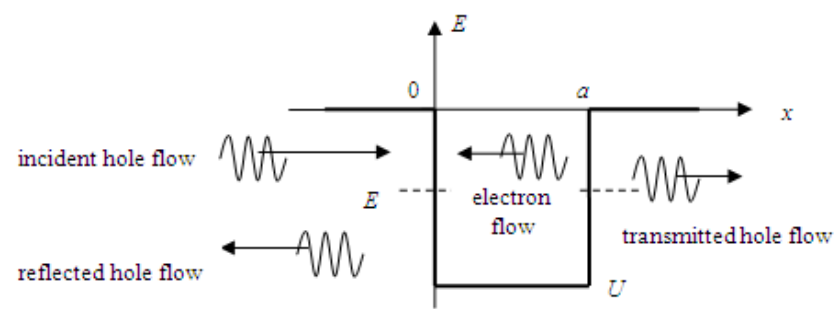

Figure 3. Schematic illustration of hole Klein tunneling

$$
0<\theta<\arcsin \frac{U-E}{E}=\arcsin \frac{|U|-|E|}{|E|}
$$


The same is obviously valid for opposite $k$ or/and $p$.

\section{In Sector II of Fig. 2:}

$$
\kappa>\mathrm{k}>1-\kappa, \kappa>1 / 2,0 \leq \mathrm{k} \leq 1
$$

- Eq. (1) is now satisfied by the following set of the wave functions:

$$
\begin{aligned}
\varphi_{A}(x) & =\left\{\begin{array}{l}
e^{i p x}+r e^{-i p x}, \quad x<0 \\
b e^{\lambda x}+c e^{-\lambda x}, \quad 0<x<a \\
t e^{i p(x-a)}, \quad x>a
\end{array}\right. \\
\varphi_{B}(x) & = \begin{cases}-\frac{i}{\kappa}\left[(k-i p) e^{-i p x}+r(k+i p) e^{i p x}\right], \quad x<0 \\
+\frac{i}{\bar{\kappa}}\left[(k-\lambda) b e^{\lambda x}+(k+\lambda) c e^{-\lambda x}\right], \quad 0<x<a \\
-i \frac{k-i p}{\kappa} t e^{i p(x-a)}, \quad x>a\end{cases}
\end{aligned}
$$

where:

$$
p=\sqrt{\kappa^{2}-k^{2}}, \lambda=\sqrt{k^{2}-\bar{\kappa}^{2}} \equiv \sqrt{k^{2}-(1-\kappa)^{2}}
$$

Pay attention that in contrast to (2) the wave functions decay inside the well, nevertheless forming the net electron flow as illustrated schematically in Fig. 4.

Boundary conditions of continuity for the wave functions at $x=0$ and $x=a$ result in the set of equations for $r, b, c$ and $t$; in dimensionless variables they are:

$$
\left\{\begin{array}{l}
1+r=b+c \\
\bar{\kappa}[(k-i p)+r(k+i p)]=-\kappa[(k-\lambda) b+(k+\lambda) c] \\
b e^{\lambda \mu}+c e^{-\lambda \mu}=t \\
\kappa\left[(k-\lambda) b e^{\lambda \mu}+(k+\lambda) c e^{-\lambda \mu}=-\bar{\kappa}(k-i p) t\right.
\end{array}\right.
$$

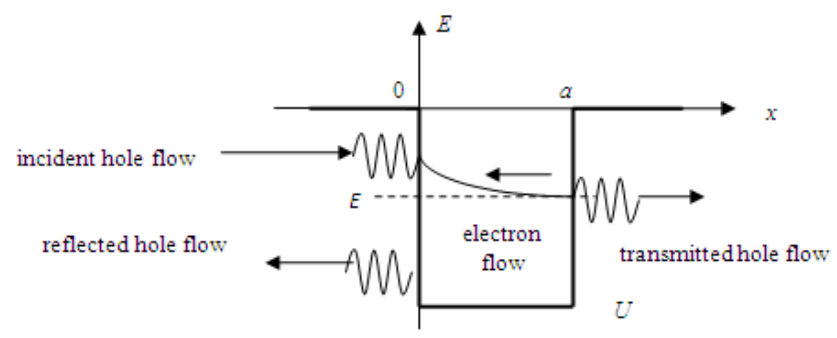

Figure 4. Schematic illustration of conventional hole tunneling

Let $\bar{\kappa}<<1, \kappa=1-\bar{\kappa} \sim 1$. Then approximately

$$
(k-\lambda) \approx \frac{\bar{\kappa}^{2}}{2 k}<<1,
$$

and we find at $\kappa \rightarrow 0$ for $\lambda \mu \gg>1$ :

$$
\begin{aligned}
& b \approx \frac{2 i p}{k-i p} e^{-2 k \mu} ; c \approx-\left.i p \frac{\bar{\kappa}}{k} \rightarrow 0\right|_{\bar{\kappa} \rightarrow 0} ; \\
& t \approx \frac{2 i p}{k-i p} e^{-\lambda \mu}, r \approx-1+\frac{2 i p}{k-i p} e^{-2 k \mu}
\end{aligned}
$$

\section{In sector III of Fig. 2:}

$$
\kappa \leq 1 / 2, \kappa \leq k \leq 1-\kappa
$$

- Eq. (1) is satisfied by the following set of the wave functions:

$$
\varphi_{A}(x)=\left\{\begin{array}{l}
A e^{\gamma x}, \quad x<-a / 2 \\
B e^{i q x}+C e^{-i q x}, \quad-a / 2<x<a / 2 \\
D e^{-\gamma x}, x>a / 2
\end{array}\right.
$$

$$
\varphi_{B}(x)= \begin{cases}-i \frac{k-\gamma}{\kappa} A e^{\gamma x}, & x<-a / 2 \\ +\frac{i}{\bar{\kappa}}\left[(k-i q) B e^{i q x}+(k+i q) C e^{-i q x}\right], \quad-a / 2<x<a / 2, \\ -i \frac{k+\gamma}{\kappa} D e^{-\gamma x}, \quad x>a / 2\end{cases}
$$

where

$$
\gamma=\sqrt{k^{2}-\kappa^{2}}, q=\sqrt{\bar{\kappa}^{2}-k^{2}} .
$$

They describe quasi-localized electron-like states which 
are localized in the $x$-direction predominantly inside the quantum well as shown schematically in Fig.5.

Matching boundary conditions:

$$
\begin{gathered}
\varphi_{A, B}(-a / 2-0)=\varphi_{A, B}(-a / 2+0), \\
\varphi_{A, B}(a / 2-0)=\varphi_{A, B}(a / 2+0)
\end{gathered}
$$

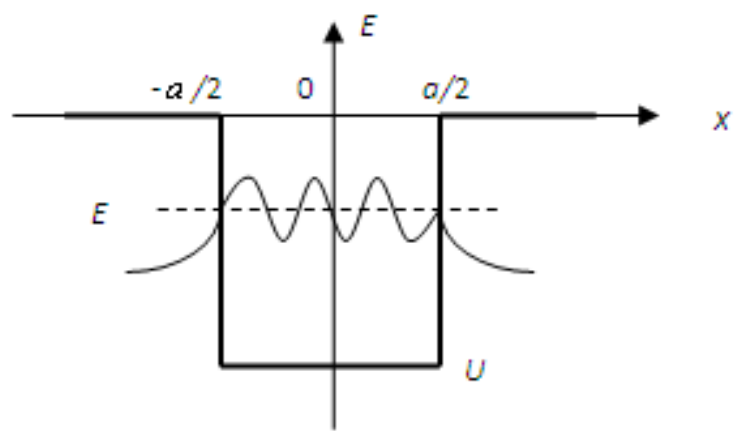

Figure 5. Schematic presentation of a localized state in the QW gives:

$$
\left\{\begin{array}{l}
\left(\frac{k-\gamma}{\kappa}+\frac{k-i q}{\bar{\kappa}}\right) B e^{-i q a / 2}+\left(\frac{k-\gamma}{\kappa}+\frac{k+i q}{\bar{\kappa}}\right) C e^{+i q a / 2}=0 \\
\left(\frac{k+\gamma}{\kappa}+\frac{k-i q}{\bar{\kappa}}\right) B e^{+i q a / 2}+\left(\frac{k+\gamma}{\kappa}+\frac{k+i q}{\bar{\kappa}}\right) C e^{-i q a / 2}=0
\end{array},\right.
$$

which, for non-zero $B$ and $C$ solution, leads to the equation for $\kappa$, i.e. for the energy of electron-like states localized in the quantum well at given $k$ (in dimensionless form):

$$
\frac{\kappa(1-\kappa)+k^{2}}{\gamma q}=-\operatorname{ctg} \mu q,
$$

or equivalently:

$$
\frac{\gamma q}{\kappa(1-\kappa)+k^{2}}=-\operatorname{tg} \mu q
$$

First of all we draw one's attention that such states may exist for normalized $\kappa \leq 1 / 2$ only (see Fig. 2) - that is for energies no lower than a half of QW depth: $|E|<|U| / 2$, and with $y$-component of the momentum

$$
k<k_{0}=\mu / a=|U| / \hbar v_{0} .
$$

Note also that the obvious solution $q=0$ does not imply any electron state as in that case $B=-C$ in (9) due to the boundary conditions and consequently $\varphi_{A}=\varphi_{B}=0$.
One can easily see that the left side of Eq. (10) is positive in the whole square $0 \leq \kappa \leq 1,0 \leq k \leq 1$ of Fig. 2. However the right side of Eq. (10) is positive only if $(2 n-1) \pi / 2<\mu q<\pi n$ for some integer $n$.

With that in mind one concludes that Eq. (11) may have solutions only if

$$
(2 n-1)^{2} \pi^{2} / 4 \mu^{2} \leq(1-\kappa)^{2}-k^{2}<\pi^{2} n^{2} / \mu^{2},
$$

i.e. in the domain between to hyperbolas inside sector $I I I$ of Fig 2:

$$
\frac{(1-\kappa)^{2}}{\pi^{2} n^{2} / \mu^{2}}-\frac{k^{2}}{\pi^{2} n^{2} / \mu^{2}}=1,
$$

and

$$
\frac{(1-\kappa)^{2}}{(2 n-1)^{2} \pi^{2} / 4 \mu^{2}}-\frac{k^{2}}{(2 n-1)^{2} \pi^{2} / 4 \mu^{2}}=1 .
$$

As follows from above, a solution of (10) may exist provided $\mu q>\frac{\pi}{2}$. As $q<1$, there is no solution of (10) when $\mu<\frac{\pi}{2}$.

Let's now consider the states $\kappa=0$. In that case we have for normalized values:

$$
\bar{\kappa}=1 ; \gamma=k ; q=\sqrt{1-k^{2}} ; k=\sqrt{1-q^{2}},
$$

and for $k \neq 0$ Eq. 10.1 reduces to

$$
q=-\sqrt{1-q^{2}} \operatorname{tg} \mu q
$$

Put $\mu q=z, q=z / \mu$; then the above equation turns to:

$$
-\frac{\sqrt{\mu^{2}-z^{2}}}{z}=\operatorname{ctg} z
$$

or

$$
-\frac{z}{\sqrt{\mu^{2}-z^{2}}}=\operatorname{tg} z
$$

Graphical interpretation of those equations is presented below in Fig. 6 (alb). One can see that there is no other solution for $0 \leq \mu<\pi / 2$ but $\kappa=0, k=1(q=z / \mu=0)$. However for $\mu \geq \pi / 2$ new solutions appear: particularly, there are $n$ other solutions $k=k_{i}$ for a given $\mu$ if

$$
(2 n-1) \pi / 2 \leq \mu<(2 n+1) \pi / 2,(n=1,2, \ldots) .
$$




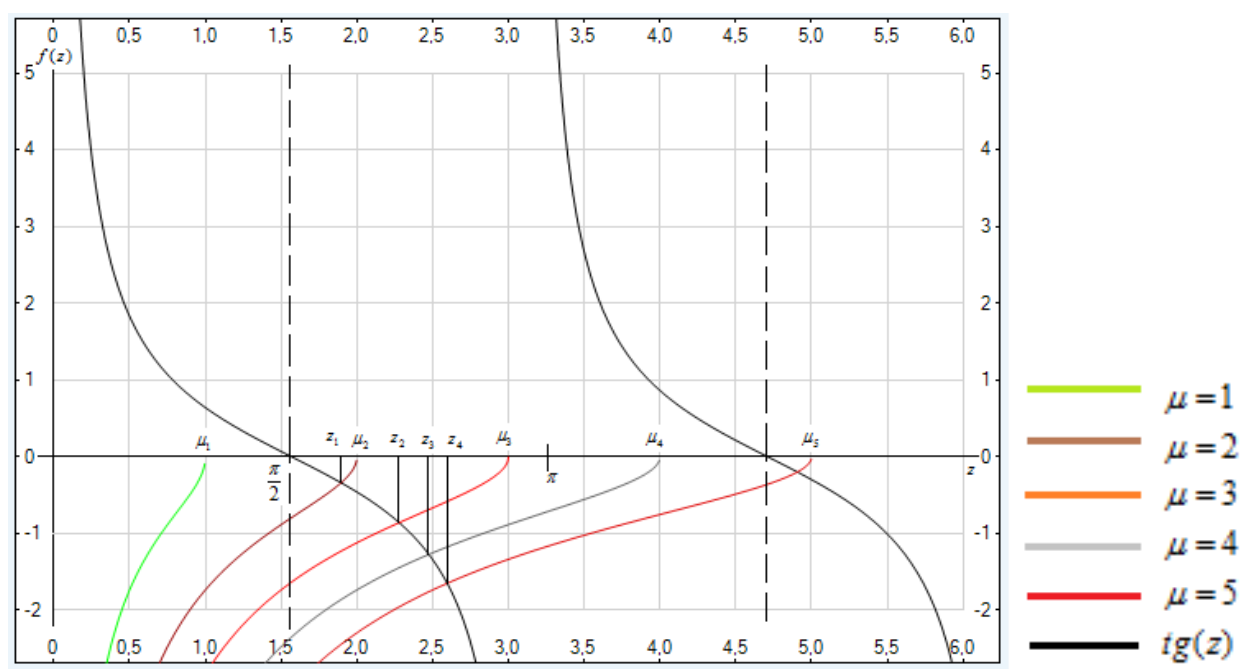

Figure 6a. Graphical solutions of Eq. (13.1)

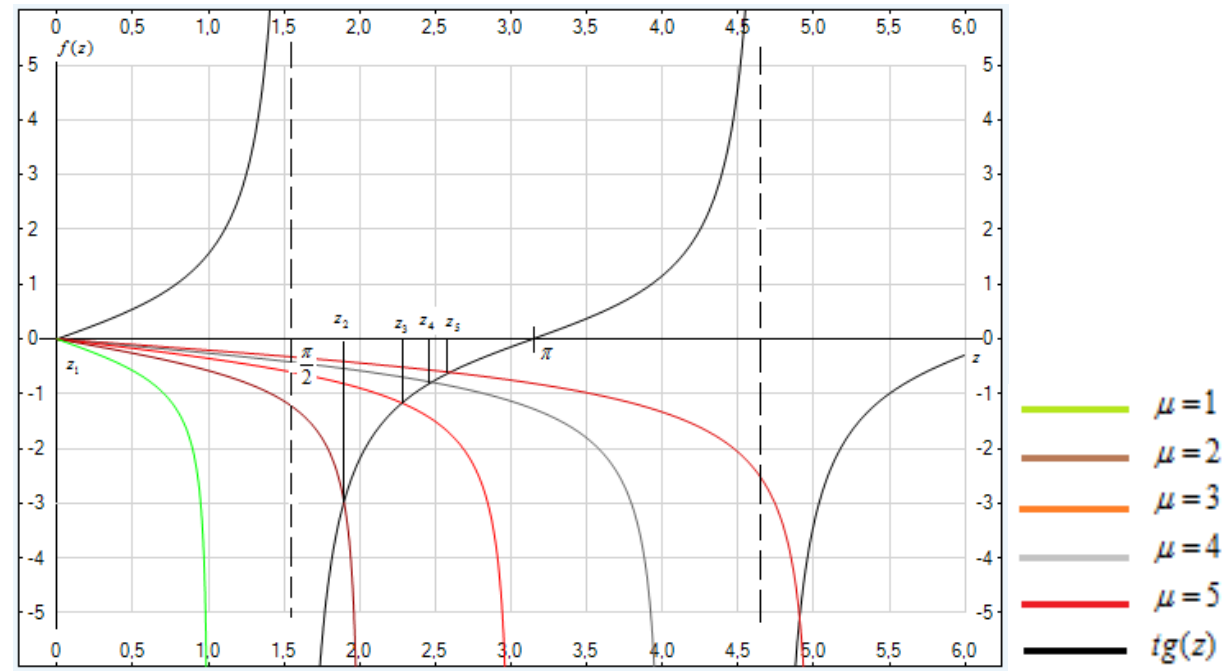

Figure 6b. Graphical solutions of Eq. (13.1) - a and Eq. (13.2) - b

They are:

$$
\kappa=0, \bar{\kappa}=1 ; q=z_{i} / \mu ; k=k_{i}=\sqrt{1-\left(z_{i} / \mu\right)^{2}} ; \gamma=\gamma_{i}=k_{i},
$$

where $z_{i} \leq \mu(i=1,2, \ldots, n)$ are the solutions of Eq. (13.1) or (13.2). We draw one's attention to the fact that the electron in such a state with zero energy has non-zero momentum:

$$
\hbar k_{i}=\hbar \frac{\mu}{a} \sqrt{1-\left(z_{i} / \mu\right)^{2}}=\frac{|U|}{v_{0}} \cdot \sqrt{1-z_{i}^{2}\left(\frac{\hbar v_{0}}{|U| a}\right)^{2}} .
$$

Evidently there is no solution $0 \leq \kappa(k) \leq 1 / 2, \quad 0<k \leq 1$ for $\pi / 2 \leq \mu<\pi$ but the above found (14). That is because for $\kappa>0$ the left side of Eq. (10.1):

$$
\frac{\kappa(1-\kappa)+k^{2}}{\gamma q}=\frac{(1-\kappa)-q^{2}}{q \sqrt{(1-2 \kappa)-q^{2}}}
$$

tends to infinity when $q^{2}$ verge towards $(1-2 \kappa)$ and does not exist when $q^{2}>(1-2 \kappa)$ : obviously, possible $q$ values can't exceed $\sqrt{1-2 \kappa}$ for given $\kappa$ with $0<\kappa<1 / 2$. So the curves representing the left and right side of Eqs. (13) may intersect only when $\mu \sqrt{(1-2 \kappa)}>\pi$ which is possible if $\mu>\pi$.

Consider now the extreme case of states with $\kappa=k \leq 1 / 2$. In that case we have for normalized values:

$$
\kappa=k \leq \frac{1}{2} \Rightarrow \bar{\kappa}=1-\kappa=1-k ; q=\sqrt{1-2 k} ; \gamma=0,
$$

and Eq. (10.1) reduces to:

$$
\operatorname{tg} \mu \sqrt{1-2 k}=0
$$

its solutions are:

$$
k=k_{m}=\frac{1}{2}\left(1-\frac{\pi^{2} m^{2}}{\mu^{2}}\right), m=1, \ldots, m<\frac{\mu}{\pi}
$$


(we omit $m=0$ as $q=0$ in that case - see comment above).

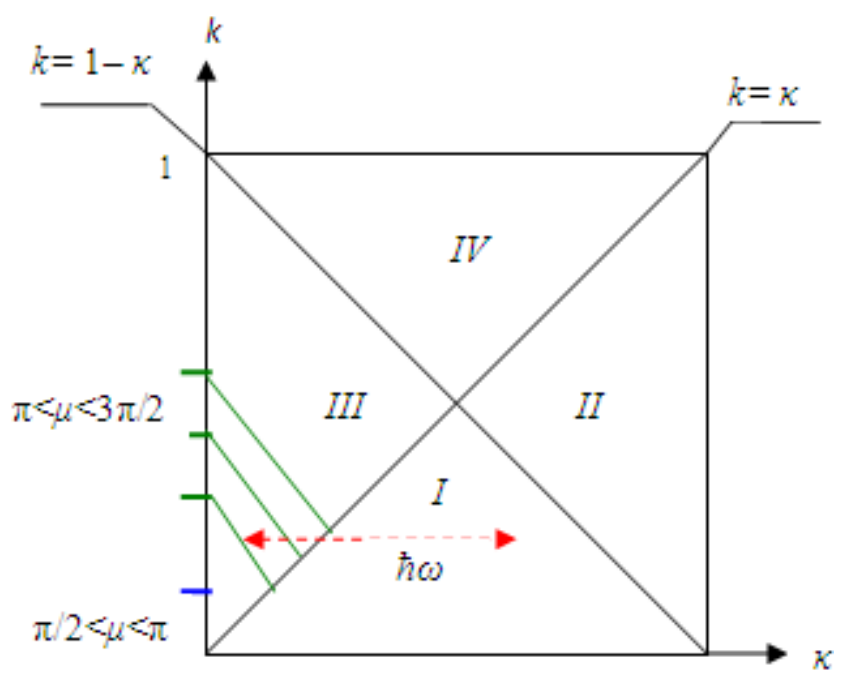

0

1

Figure 7. Schematic representation of localized excitation spectra for two QW of different power for increasing $\mu$

One can see from (16) that solutions

$$
\kappa=k, 0<\kappa \leq 1 / 2, \kappa=k, 0<k \leq 1
$$

appear only when $\mu>\pi$, as was discussed above. Actually the continuous brunches $0<\kappa=\kappa(k) \leq k$ appear in that case: the first one (and the only one as yet) - when $\pi<\mu<3 \pi / 2$. They start at $k=0$ from $k(0)=k_{i}$ from (14) and end at $k=k_{m}$ from (16) with $i=m$; evidently $k(0)>k_{m}$, so one can suggest that the energy spectrum is of the kind shown schematically in Fig. 7 for some values of $\mu$.

When $\mu$ exceeds $3 \pi / 2$ new spectral branch appear similar to described above, while the first one goes up closely to the line $k=1-\kappa$. So on, each time $\mu$ exceeds $(2 n+1) \pi / 2$, one more spectral branch of that kind emerges.

Sector IV of Fig. 2: $k>\kappa, k>1-\kappa, k>1 / 2,0 \leq \kappa \leq 1$.

Eq. (1) is now satisfied by the following set of the wave functions:

$$
\begin{aligned}
& \varphi_{A}(x)=\left\{\begin{array}{l}
A e^{\gamma x}, \quad x<-a / 2 \\
B e^{\alpha x}+C e^{-\alpha x}, \quad-a / 2<x<a / 2 \\
D e^{-\gamma x}, x>a / 2
\end{array}\right. \\
& \varphi_{B}(x)=\left\{\begin{array}{l}
-i \frac{k-\gamma}{\kappa} A e^{\gamma x}, \quad x<-a / 2 \\
\frac{i}{\bar{\kappa}}\left[(k-\alpha) B e^{\alpha x}+(k+\alpha) C e^{-\alpha x}\right],-a / 2<x<a / 2, \\
-i \frac{k+\gamma}{\kappa} D e^{-\gamma x}, \quad x>a / 2
\end{array}\right.
\end{aligned}
$$

where $\gamma=\sqrt{k^{2}-\kappa^{2}} ; \alpha=\sqrt{k^{2}-\bar{\kappa}^{2}}$.

Such electron states, if exist, are localized near the walls of the quantum well.
Matching boundary conditions:

$$
\begin{gathered}
\varphi_{A, B}(-a / 2-0)=\varphi_{A, B}(-a / 2+0) \\
\varphi_{A, B}(a / 2-0)=\varphi_{A, B}(a / 2+0)
\end{gathered}
$$

leads now to the following equation for $\kappa$, i.e. for the allowed energy, if any, of such "electron-hole" states at QW borders

(in dimensionless form):

$$
\kappa(1-\kappa)+k^{2}=-\gamma \alpha \operatorname{coth} \alpha \mu .
$$

The left side of eq. (18) is always positive, in domain $I V$, while the right side is always negative. So eq. (18) has the only solution $\alpha=0$, which however does not imply any electron state as $B=-C$ in that case and consequently $\varphi_{A}=$ $\varphi_{B}=0$ like above in section III. The system has no allowed electron states for $k$ and $\kappa$ in section IV - so the states (17) localized near the walls of the quantum well do not exist.

\section{Conclusions}

We have studied quantum electron states in free-standing graphene monolayer with a rectangular quantum well. We have shown that alongside with delocalized Klein tunneling states, described elsewhere earlier, 1D elementary excitations, localized mainly in the quantum well, may exist in such structure if the QW potential has enough power. The energy of such states lies no deeper than a half of the QW depth. They form a sort of a peculiar quantum "rod" in a grapheme monolayer. For deeper states conventional hole tunneling may take place. While the excitations' momentum absolute value along the QW for Klein tunneling states cannot exceed

$$
|U| / 2 \hbar v_{0},
$$

localized electrons can possess any momentum in that direction:

$$
-|U| / \hbar v_{0}<k<|U| / \hbar v_{0},
$$

as well as conventionally tunneling holes do. Radiative transitions can take place between those states with the same $k$ as shown in Fig. 7. Similar scenario is obviously realized for hole-like states under a potential barrier.

\section{Acknowledgements}

The work is supported by the Russian Foundation for Basic Research, Project No. 14-01-00663-a.

\section{REFERENCES}

[1] K. S. Novoselov, A. R. Geim., S. V. Morozov, D. Jiang, Y. Zhang, S. V. Dubonos, I. V. Grigorieva, A. A. Firsov. Electric Field Effect in Atomically Thin Carbon Films, 
Science, Vol. 306, 666-969, 2004.

[2] K. S. Novoselov, A. K. Geim, S. V. Morozov, D. Jiang, M. I. Katsnelson, I. V. Grigorieva, S. V. Dubonos and A. A. Firsov. Two-dimensional gas of massless Dirac fermions in graphene, Nature, Vol. 438, 197-200, 2005.

[3] A. K. Geim. Graphene: Status and Prospects, Science, Vol. 324, 1530-1534, 2009.

[4] A. K. Geim and K. S. Novoselov. The rise of graphene, Nature Materials, Vol. 6, 183 - 191, 2007.

[5] O. Klein. Die Reflexion von Elektronen an einem Potentialsprung nach der relativistischen Dynamik von Dirac, Zeitschrift für Physik, Vol. 53, No. 3-4, 157-165, 1929.

[6] Cheianov, V.V., and V.I. Fal'ko. Selective transmission of Dirac electrons and ballistic magnetoresistance of $n-p$ junctions in graphene, Phys. Rev., Vol. B74, 041403(R), 2006.

[7] M. I. Katsnelson, K. S. Novoselov, and A. R. Geim. Chiral tunnelling and the Klein paradox in graphene, Natur. Phys, Vol. 2, No. 9, 620-625, 2006.

[8] A. H. Castro Neto, F. Guinea, N. M. R. Peres, K. S. Novoselov, and A. R. Geim. The electronic properties of grapheme, Rev. Mod. Phys., Vol. 81, 109-162, 2009.
[9] C. Bai and X. Zhang. Klein paradox and resonant tunneling in a graphene superlattice, Phys. Rev. B, Vol. 76, 075430-075436, 2007.

[10] H. V. Nguyen and V. L. Nguyen. Tunneling of Dirac electrons trough one-dimensional potentials in graphene: a T-matrix approach, J. Phys.: C, Vol. 21, 045305-045310, 2009.

[11] X. Xu, G. Xu and J. Cao. Electron tunneling trough a trapezoidal barrier in graphene, Japan. Journ. Appl. Phys., 085201-085204, 2010.

[12] V.A.Fedirko, Electron Tunneling from Graphene, UJPA, Vol. 2(6): 263-267, 2014, online available from http://www.hrpub.org

[13] McClure, J. W. Diamagnetism of graphite., Phys. Rev., Vol. 104, 666-671, 1956

[14] Semenoff, G. W. Condensed-matter simulation of a three-dimensional anomaly, Phys. Rev. Lett., Vol. 53, 24492452, 1984.

[15] DiVincenzo, D. P., and E. J. Mele. Self-consistent effective-mass theory for intralayer screening in graphite intercalation compounds, Phys. Rev., Vol. B 29 1685, 1984.

[16] C. W. J. Beenakker. Colloquium: Andreev reflection and Klein tunneling in graphene, Rev. Mod. Phys., Vol. 80, No.4, 1337-1354, 2008 (arXiv:0710.3848v2 [cond-mat.mes-hall]). 